\title{
Heartbeat: guidelines versus reality for patients with severe
}

doi:10.1136/heartjnl-2019-316074

The increasing prevalence of aortic stenosis (AS) in our ageing population and clinical trials demonstrating the benefits of transcatheter valve implantation for severe symptomatic AS have focused attention on this disease in recent years. Yet, despite clear recommendations from professional societies, it remains unclear whether all patients receive timely and appropriate treatment. To address this concern, Frey and colleagues ${ }^{1}$ looked at data from the Study to Improve Outcomes in Aortic Stenosis (IMPULSE) registry which included 2171 adults with severe AS at 23 tertiary care hospitals in 9 European countries, including the UK. Patient mean age was 78 years, $48 \%$ were women and $27 \%$ had a left ventricular ejection fraction less than $50 \%$. Over $80 \%$ of these patients were symptomatic, but only $76 \%$ of those with severe symptomatic AS were treated appropriately with aortic valve replacement (AVR), most often by the transcatheter approach (figure 1). Of even more concern, among asymptomatic patients with an indication for AVR, 42\% (22/52) were not treated, whereas AVR was performed in 36\% (123/339) of asymptomatic AS patients with no established indication.

In the accompanying editorial, Chambers $^{2}$ discusses the study design and comments that 'the IMPULSE registry is a useful 'snapshot' of current care and reminds us that the key to improving care for patients with valve disease is to develop a comprehensive valve service at three levels: 1 . the detection of valve disease; 2. referral of patients with moderate or severe disease to a specialist valve clinic to plan management and follow-up until intervention is indicated and 3. intervention in a heart valve centre with recognised performance standards' (figure 2).

High-sensitivity cardiac troponin testing is integral for diagnosis of acute coronary syndromes (ACSs) but the potential value of troponin measurements months to years after the acute event has not been studied. In this issue of Heart, Adamson and colleagues ${ }^{3}$ found that troponin

Correspondence to Professor Catherine M Otto, Division of Cardiology, University of Washington, Seattle, WA 98195, USA; cmotto@uw.edu aortic stenosis

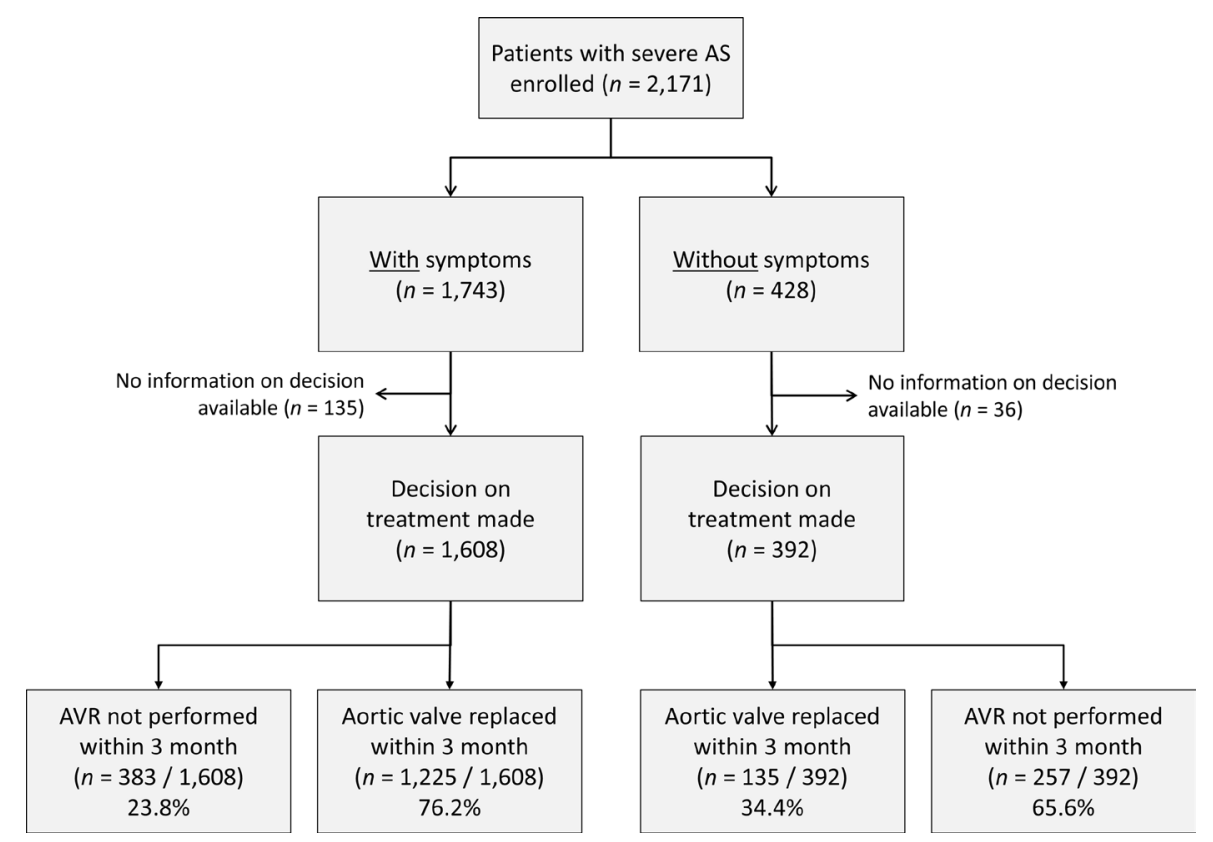

Figure 1 Flowchart of the population and management according to functional status. AS, aortic stenosis; AVR, aortic valve replacement. concentrations 4 months after an ACS were an independent predictor of cardiovascular death, with an HR of 1.4 (CI 1.3 to 1.5$)$ per doubling of the serum level (figure 3). Risk was highest in patients who had increasing troponin levels at 12 months and in those with a 4-month level $>$ 99th percentile compared those with a troponin $\leq 5 \mathrm{ng} / \mathrm{L}(29.5 \%(49 / 166)$ vs
4.3\% (34/795); adjusted HR 4.9, 95\% CI 3.8 to 23.7$)$.

In a provocative editorial, Kavask and Devereaux ${ }^{4}$ commend the authors for 'undertaking and executing this study, laying the foundation for a possible testing protocol with high-sensitivity cardiac troponin following ACS.' However, in the context of varying definitions, methods
Figure 2 The valve disease network. AF, atrial fibrillation; EP, electrophysiologic; ITU, intensive treatment unit; TAVI, transcatheter aortic valve implantation. 


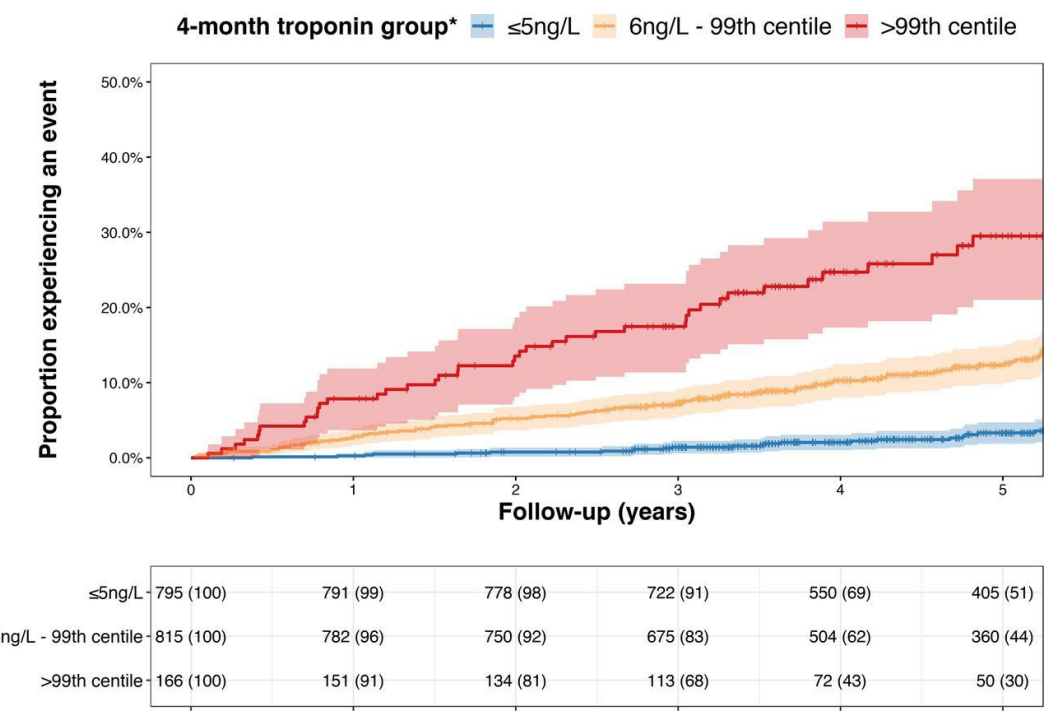

Figure 3 Cumulative incidence of cardiovascular death according to 4-month troponin concentration cumulative event curves for cardiovascular death according to troponin concentrations determined at the 4-month visit. Each cross-hair indicates when a subject is censored from further follow-up. The number at risk (\% in group remaining) for each yearly interval is given for each troponin group. Follow-up begins from date of 4-month visit. *For descriptive purposes, troponin concentrations have been rounded to nearest integer value. Therefore $\leq 5 \mathrm{ng} / \mathrm{L}$ includes all patients $<5.5 \mathrm{ng} / \mathrm{L}$.

and variability in high-sensitivity troponin testing, they point out that 'a question lingers as to whether current laboratory practices can provide accurate and reproducible results around the thresholds of such a framework.' Based on a simulation using their own quality control data 'that the percent change criterion as suggested by Adamson and colleagues ${ }^{3}$ would be appropriate for concentrations near the 99th percentile but not at the lower concentration limit of $6 \mathrm{ng} / \mathrm{L}$, where absolute changes (rather than percent) in for troponin measurements, they suggest high-sensitivity cardiac troponin at this low concentration range have demonstrated clinical utility.'

The importance of reporting and analysing sex difference in cardiovascular outcomes is discussed in detail in a review article and tutorial ${ }^{5}$ by Woodward in this issue of Heart. Readers will find that this article provides a clear rationale for the optimal approaches to analysing sex differences with examples shown in figures and tables. Clinical researchers will find this article is a practical guide to study design and data analysis with a table (table 1) of specific recommendations for reporting study results.

Our Education in Heart article reviews clinical indications for cardiovascular magnetic resonance (CMR) imaging ${ }^{6}$ with tables highlighting the strengths of different imaging techniques and detailing CMR findings that differentiate restrictive and hypertrophic cardiomyopathies (figure 4).

In our Cochrane Corner series, short summaries of recent Cochrane Reviews relevant to clinical cardiology, Stranges and colleagues ${ }^{7}$ address the question 'Does the Mediterranean-style diet help in the prevention of cardiovascular disease?' They conclude: 'At the present time, there is no definitive trial evidence regarding the effects of a Mediterranean-style diet on clinical endpoints for both the

\begin{tabular}{|c|c|}
\hline \multicolumn{2}{|c|}{ General } \\
\hline G1 & Consider whether the research is concerned with sex (biological) or gender (behavioural) differences, and report the results accordingly. ${ }^{*}$ \\
\hline G2 & $\begin{array}{l}\text { Routinely provide sex-disaggregated results when reporting research on cardiovascular associations. This includes prespecifying subgroup analyses by sex. When } \\
\text { there are no important sex differences, still include sex-specific results, most likely in the appendix of a manuscript for publication. }\end{array}$ \\
\hline G3 & Even when a study is concerned with associations for a single sex, where possible compare results for the other sex, as a control. \\
\hline G4 & Adjust at least for age when comparing sex-specific cardiovascular associations. \\
\hline G5 & $\begin{array}{l}\text { Consider analyses on both the relative and absolute scales. When it is only appropriate to present relative risks, provide (at least) the number of events and the } \\
\text { number at risk across the sex by risk factor exposure cross-classes, to give context to the reader. }\end{array}$ \\
\hline G6 & Quantify the sex difference (with accompanying measure of uncertainty, such as a 95\% Cl), rather than merely test for a significant difference. \\
\hline G7 & $\begin{array}{l}\text { When analysing raw (ie, individual participant) data, use the full interaction model (with all main effects and two-way interactions) to obtain the sex-specific results } \\
\text { as well as the sex comparison(s). }\end{array}$ \\
\hline G8 & Unless there is statistical or clinical significance in the sex difference (ie, the sex interaction), avoid sex-specific conclusions. \\
\hline \multicolumn{2}{|r|}{ Specific to meta-analyses } \\
\hline M1 & Decide whether to use the fixed effect or random effects method before data are collected. \\
\hline M2 & Only include studies with results from both sexes. \\
\hline M3 & In the report, include a flow chart with reasons for exclusions. Clearly state the number of studies excluded for want of sex-disaggregated results. \\
\hline M4 & Use reliable, general, statistical softwaret, such as R or Stata. \\
\hline M5 & $\begin{array}{l}\text { Include forest plots by sex and to compare the sexesł. Show age-adjusted and multiple-adjusted analyses separately, where appropriate. This will typically require } \\
\text { placing some forest plots in the appendix of a manuscript for publication. }\end{array}$ \\
\hline M6 & $\begin{array}{l}\text { Following the meta-analysis, use meta-regression and bubble plots to explore sources of heterogeneity, to include overall risk and the difference between the sex- } \\
\text { specific risks. }\end{array}$ \\
\hline M7 & $\begin{array}{l}\text { Take care when pooling ORs together with relative risks or HRs. Stratify pooling by the metric used where risk (or, in cross-sectional studies, prevalence) is typically } \\
\text { high. }\end{array}$ \\
\hline
\end{tabular}

*In this manuscript no distinction is made, for simplicity of exposition.

tThese have the advantage of offering a wide range of other tools, so that the extra work of learning the basics of such a package (if necessary) will be worthwhile. ‡For example, through the ratio of relative risks-see figure 2 . 


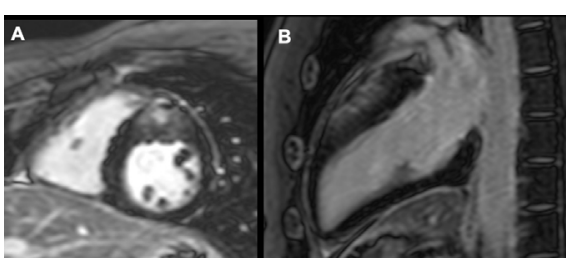

Figure 4 Patient with hypertrophic cardiomyopathy with asymmetric anterior and anteroseptal hypertrophy and an associated non-ischaemic diffuse scar in the area of maximal hypertrophy $(A, B)$.

primary and secondary prevention of major CVD. Overall, the available trial evidence is promising (though not conclusive) and generally supportive of favourable effects of the Mediterranean-style diet on individual cardiometabolic risk factors in primary prevention studies, and potentially also on clinical endpoints such as stroke. Several ongoing trials, particularly those reporting clinical endpoints in secondary prevention, will add to the evidence base.'

Funding The authors have not declared a specific grant for this research from any funding agency in the public, commercial or not-for-profit sectors.

Competing interests None declared.

Patient consent for publication Not required.

Provenance and peer review Commissioned; internally peer reviewed.

(c) Author(s) (or their employer(s)) 2019. No commercial re-use. See rights and permissions. Published by BMJ.

\section{(A) Check for updates}

To cite Otto CM. Heart 2019:105:1683-1685.

Heart 2019:105:1683-1685.

doi:10.1136/heartjnl-2019-316074
ORCID iD

Catherine M Otto http://orcid.org/0000-0002-05279392

\section{REFERENCES}

1 Frey N, Steeds RP, Rudolph TK, et al. Symptoms, disease severity and treatment of adults with a new diagnosis of severe aortic stenosis. Heart 2019:105:1709-16.

2 Chambers JB. Aortic stenosis: service delivery before guidelines. Heart 2019;105:1686-7.

3 Adamson PD, McAllister D, Pilbrow A, et al. Convalescent troponin and cardiovascular death following acute coronary syndrome. Heart 2019;105:1717-24.

4 Kavsak P, Devereaux PJ. High-Sensitivity troponin testing months after an acute coronary syndrome: noise or notable results. Heart 2019:105:1688-90.

5 Woodward M. Rationale and tutorial for analysing and reporting sex differences in cardiovascular associations. Heart 2019;105:1701-8.

6 Nagel E. Clinical indications for cardiovascular magnetic resonance. Heart 2019:105:1755-62.

7 Stranges S, Takeda A, Martin N, et al. Cochrane corner: does the Mediterranean-style diet help in the prevention of cardiovascular disease? Heart 2019;105:1691-4. 